\title{
Visible light communications: application to cooperation between vehicles and road infrastructures
}

\author{
A. Cailean, B. Cagneau, L. Chassagne, S. Topsu, Y. Alayli and J-M. Blosseville
}

\begin{abstract}
Since a few years, the vehicle industry tends to increase the performances of the lights based on led technologies. Nowadays, led systems are used as a standard by motor vehicles manufacturers. Led lights present higher reliability and are more flexible regarding the design or power adjustments. Furthermore, led systems are also very convenient for intensity modulation like in telecommunication fields. We developed a very simple data transmission system based on led that aims to be highly robust at short or medium distances - a few meters up to 15 meters. This visible light communication is dedicated to the cooperation between vehicles and road infrastructures to enhance the security of the traffic. This paper explains the choices and first results on the data transmission performances. In this paper, we present a first prototype of the system we made and experimental results.
\end{abstract}

\section{INTRODUCTION}

$S^{\mathrm{I}}$ NCE a few years, the automobile industry greatly improved vehicles safety and comfort with more and more intelligence, sensors and instrumented systems in order to sense environmental conditions and adapt their behaviors. Cooperation between vehicles or with road infrastructures is a key point to enhance the efficiency and safety in heavy density traffic in motorway or in suburbs configurations. The more the vehicles can sense their environment and communicate each-other, the more the traffic behavior can be sensed and then improved. Nowadays, a lot of assistance helping systems can alert the driver, and even act with quasiautonomous control. One can expect that near future vehicle generations will be fully automated.

Since many years, laboratories and car industry are very concerned by autonomous vehicles. However, due to technical limits and law enforcement, automation did not succeed as expected.

Nevertheless the legal background is now changing and possibilities of fully automated vehicles are authorized in certain circumstances. Researchers are mainly working on two different fields. The first one basically concerns the behavior and the control of the vehicle. Platooning, as

This work was supported in part by the University of Versailles SaintQuentin and Valeo Industry.

All authors except J-M Blosseville work at the University of Versailles Saint-Quentin, 45 avenue des Etats-Unis 78035 Versailles France (corresponding author phone: $+33 \quad 1 \quad 39 \quad 25 \quad 49$ 44; $\quad$ e-mail: barthelemy.cagneau@ uvsq.fr).

J-M. Blosseville works at IFSTTAR, French Institute of Science and Technology for Transport, Development and Networks, 25 allée des marronniers, $\quad 78000 \quad$ Versailles-Satory, France, (jeanmarc.blosseville@inrets.fr).

A part of the financial support is granted by the Fond Unique Interminsteriel (FUI) project named Co-Drive, supported by the Pôle de Compétitivité Mov’eo. explained in [1], is a perfect example of this purpose. It explains how the stability of the whole system is closely linked to the number of vehicles and to the models used to synthesize the controller. Control laws and signal processing are thus an important issue in order to guarantee the stability but also to ensure high-speed data transfer and communication [2]. As far as platooning and traffic control are concerned, path planning algorithms are of primary interest [3-4]. Complex models and sophisticated control laws are needed to control the vehicles. Moreover, path planning and virtual links between the vehicles are also a key point to improve the traffic through the behavior of the motorized vehicles.

The second field of research relates to the perception of the environment. It is not only useful to derive robust control schemes but also because of the need of sensors to interact with the environment. Dedicated sensors, such as the prototype presented in this paper or in, are a prerequisite to enhance the communication between the vehicles but also with the infrastructures [5-9].

The transmissions of data between vehicles are crucial to communicate information concerning the state of the vehicle (brake, speed, acceleration, engine failure, etc), or traffic information (state of traffic-lights, accidents, traffic jams, line works, etc) [10-11]. Data fusion is useful to adapt the behavior of the vehicles, road-lights and infrastructures for safety and traffic control.

A few consortiums [12-14] are working on automated vehicles or instrumented roads to develop automation. The most common research orientation is based on autonomous systems. In this approach, perception solutions consist in radar, lidar or camera vision systems. The camera is used to detect white lines on the roads since, radar or lidar to detect other objects on the road. All the perception systems are used to control vehicle relative position and trajectory to achieve centimetric accuracy. The future challenge is to develop communications with high bandwidth to decrease the perception phase duration.

In this paper, we will present a prototype that aims to transmit data through different channels. In section II, we will focus on visible light communication technology. In section III, the experimental setup and the core of the system are presented with simulation results. First experimental results are finally discussed in section IV. 


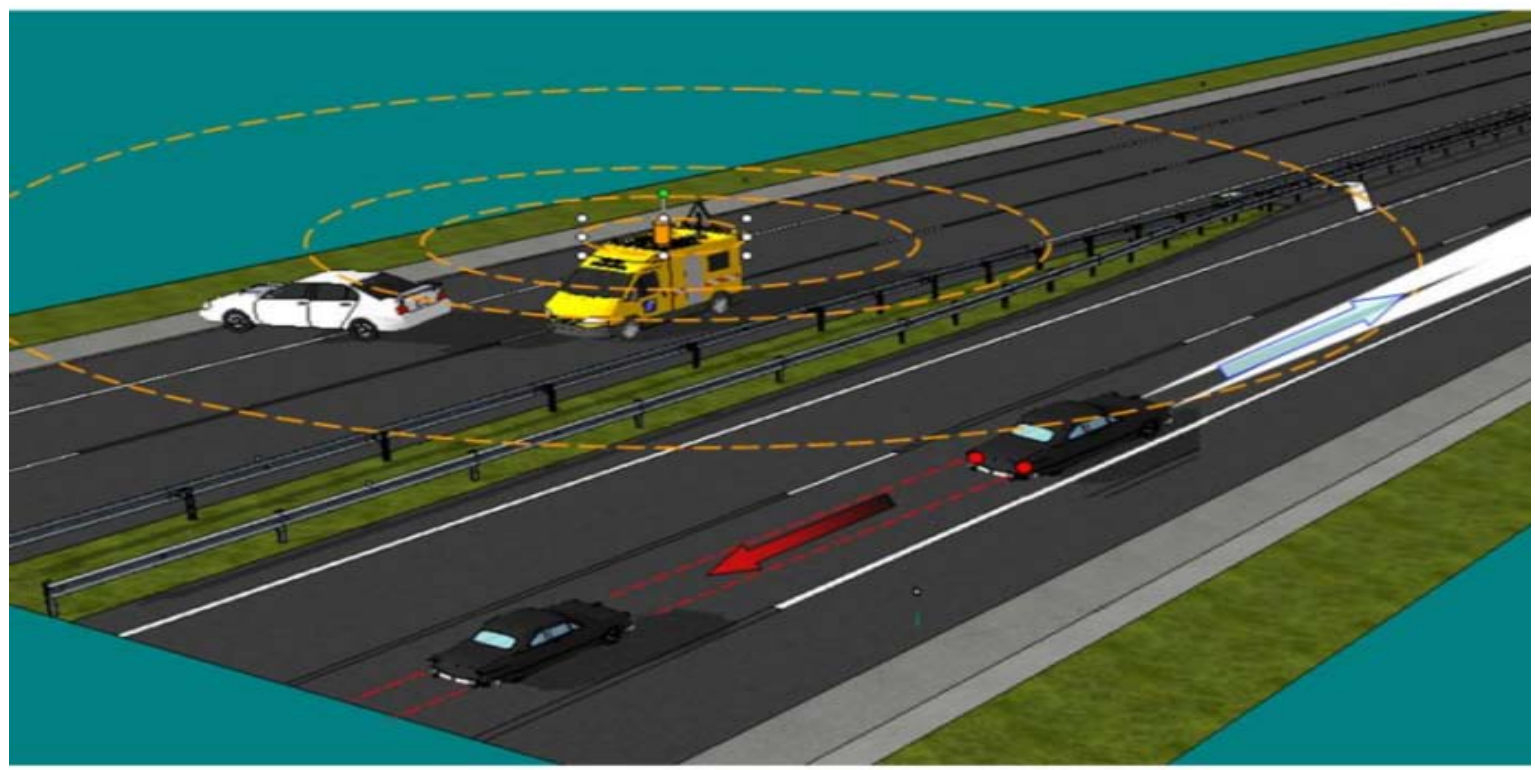

Figure 1: Illustrations of visible light communication between vehicles

\section{VISIBLE LIGHT COMMUNICATIONS BETWEEN VEHICLES}

Several methods are examined for the communications between vehicles or infrastructures. The main one is based on radiofrequency waves. Developments in wi-fi, bluetooth or radio-mobile networks have proven the efficiency of such technologies. The range of perception is tens or even hundreds of meters and can be sufficient enough for road configurations. Most of the applications need only few tens of meter ranges. Another advantage is the multidirectional propagation that can ensure a broad diffusion of the information. Nevertheless, these technologies are not possible for high speed traffic because of synchronization limits on the data frames. Furthermore, bandwidths of radiofrequency networks are often saturated and the extension of new available carriers is expensive or even impossible.

With the development of the vehicle lights, a competitive way to transmit information with high data rate is made possible. The vehicle lights have been enhanced for position and intensity control and reliability. Lights based on Light Emitted Diode (LED) matrix appear now commonly. The interests of led-lights are numerous; the very high reliability and a long life-time are the main ones. These advantages lead the automobile industry to replace in the next future the classical halogen lamps by all-led systems. Another characteristic of leds is the capability to be current modulated for optoelectronics transmissions. Led-based power modulations are very common and the technology is well-known. Since many years now, optical communications have been developed for data transmission with high rate and bandwidth. Transmission of traffic information or mechanical-states of vehicles is then fully compatible with this led capability [16]

Figure 1 illustrates a case in a motorway configuration. A security vehicle can proceed on damaged car and communicates the situation around the accident area. One car receives the data and relays the information on its line. This information can be transmitted with the headlights but also to the followers with red back-light. Data are thus propagated on the motorway. Furthermore the cars on the same line can also communicate each-other information about their mechanical state, like speed, acceleration, braking action or other data to enhance the traffic and the security.

The light sensing parts can be either camera systems, or photodetector elements. Camera systems are useful when images or pixel applications are implemented. For example, the detection of a led traffic light with embedded high-speed camera has been demonstrated [17]. The traffic light is composed of a led matrix and the perception and the recognizing of the form on the camera can be subject to complex treatments. Good performances on BER (Bit Error Ratio) have been obtained over tens of meters. Nevertheless, the camera has to be high speed model and is actually reserved for laboratories prototypes. The car industry plans to mount low-cost cameras, like the ones available on mobile phones but the performances get worse. Furthermore, the noise performances of CCD (Charge Coupled Device) cameras are lower than for independent photo-elements.

The first works on communications between infrastructures and vehicles are mainly between traffic light and vehicles. The power of traffic light is relatively high and can then allow long distance transmissions. Photosensing elements like photodetectors are quite efficient in noise performances and can be used also on long range. Nevertheless, long-range induces small angles and directional conditions. The photosensing element must be integrated in the vehicle with an optic part to focus the light. Mechanical and optic parts must be precisely realized because the solid angles are very small. Active control of the position of the sensing element has been achieved to enhance the BER [18]. 

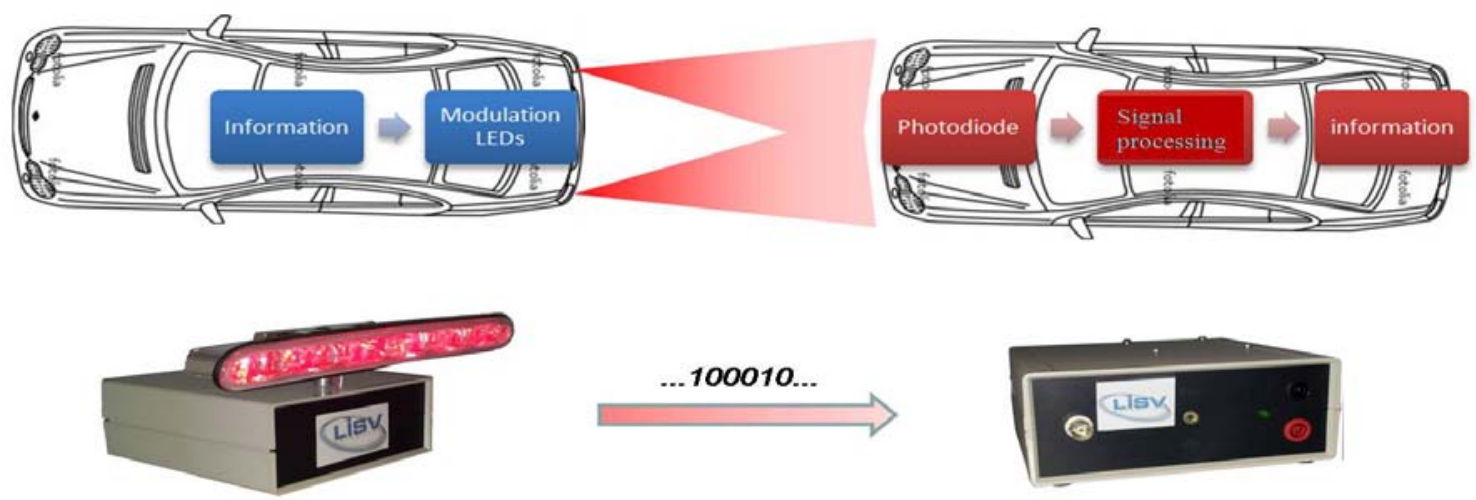

Emitter

Receiver

\begin{tabular}{|l|l|l|l|}
\hline Synchronisation & Starit & Data & Stop \\
\hline
\end{tabular}

Figure 2: First prototype of transmission data system and the associated frame of digital data.

For shorter ranges, the solid angle of emission of the light is wide enough for a passive photosensing element to be efficient without active control of the position.

\section{EXPERIMENTAL CONFIGURATION}

The LISV (Laboratoire d'Ingénierie des Systèmes de Versailles) of the University of Versailles is getting involved in a french consortium working around automated and instrumented vehicles. A patent has been deposited on optoelectronics system for communication and inter-vehicle distance measurement [19]. A prototype of led-light communications has been realized. Power modulation is used to transmit digital information illustrated on the Figure 2. At this time, the electronics is not embedded but all the components have been chosen for their low cost and their compactness.

The red back light is current modulated with OOK (On-Off Keying) amplitude modulation with a microcontroller and a cheap digital power switch. A digital frame has been defined illustrated on Figure 2. Several synchronization bits begin the frame to alert the receiving board that a message is sent. The rest of the frame is classical with start and stop bits, and an additional flag informs how long the data frame is. The clocks of the receiver is then not synchronize with phase locked-loop for simplicity, but the frequencies involved (few kilohertz, see next paragraphs) are low enough and the decoding system has no need of time accuracy. The decoding part is made online with a microcontroller that decodes the frame. Presently, the maximum data length is 96 bytes but could be longer.

\section{A. Discussion about the coding}

Two codes have been used: the biphase (Manchester code) and the Miller code. The Miller code is easily constructed from the biphase code. The power spectral densities $S_{f}$ (PSD) of these two codes are expressed by the eq. 1 and eq. 2 for Manchester and Miller code respectively:

$$
S_{\text {Man }}(f)=V^{2} T \cdot\left[\frac{\sin ^{2}(\pi f T / 2)}{\pi f T / 2}\right]^{2} \text {, }
$$

$$
\begin{gathered}
S_{\text {Mil }}(f)=\frac{V^{2} T}{2(\pi f T)^{2}[17+8 \cos (2 \pi f T)]} \\
\times \frac{1}{\left[\begin{array}{l}
23-2 \cos (\pi f T)-22 \cos (2 \pi f T)-12 \cos (3 \pi f T) \\
+5 \cos (4 \pi f T)+12 \cos (5 \pi f T)+2 \cos (6 \pi f T) \\
-8 \cos (7 \pi f T)+2 \cos (8 \pi f T)
\end{array}\right]}
\end{gathered}
$$

where $V$ is the signal amplitude and $T$ the modulation period. $f$ is the frequency for which the PSD is calculated.

The corresponding curves are plotted on Figure 3 for two examples of the modulation frequency; for clarity, the curves of the Manchester case have been inverted and are negative. The curves are proportional to a scale factor $\mathrm{K}$ depending on the amplitude of the signal but nevertheless, it is the relative scale that is interesting. 


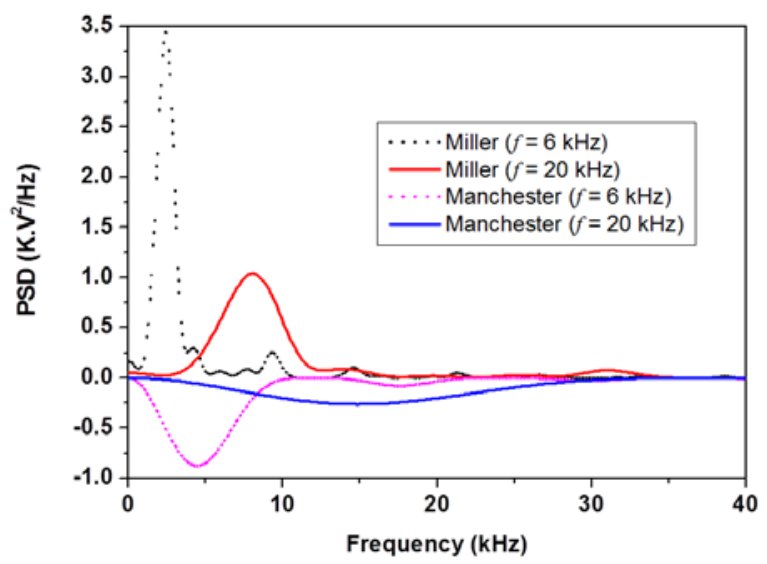

Figure 3: Comparison between Manchester and Miller PSD for two modulation frequencies. Manchester curves have been inverted and are negative for clarity.

The noticeable behavior of the Miller code is that the useful bandwidth is lower than for the Manchester code. In this example, two channels can be clearly distinguished in a total bandwidth of around $12 \mathrm{kHz}$, unless for the Manchester that requires a higher bandwidth up to $27 \mathrm{kHz}$. Hence the Figure 4 shows a five channels simulation with the Miller code, and a total bandwidth lower than $150 \mathrm{kHz}$. In Miller code PSD, the maximum is at a frequency of around 2/5 of the modulation frequency. The five sub-carriers can be easily filtered by bandpass filters, either analog, or digital.

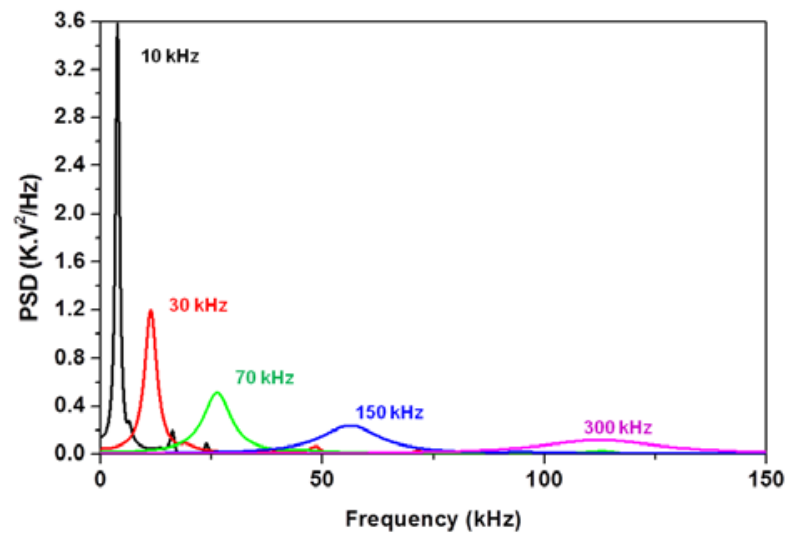

Figure 4: Simulation for a five channel configuration with Miller code at the modulation frequencies of $10 \mathrm{kHz}, 30 \mathrm{kHz}$, $70 \mathrm{kHz}, 150 \mathrm{kHz}$ and $300 \mathrm{kHz}$.

In our prototype, the microprocessor can be switched either on the Manchester code, or on the Miller code in order to test different configurations.

\section{B. Discussion about the reception module}

A low cost silicon photodetector is used, coupled with a very simple electronics. The aim of the system is to transmit over short distance, up to $15 \mathrm{~m}$ in the first version but whatever the environment conditions (rain, sun, etc). The sensitive element has been conditioned with a classical transimpedance circuit. The gain has been calculated in order not to saturate in case of direct sun exposition (100 000 lux), that has been experimentally verified. The bandwidth of the circuit is given by eq. 3 :

$$
B W=\sqrt{\frac{G B P}{2 \pi R\left(C+C_{p}\right)}}
$$

where $G B P$ is the gain-bandwidth product of the operational amplifier, $R$ the gain resistance, $C_{p}$ the capacitive part of the photodetector and $C$ the capacitive part of the amplifier. We choose elements to have a bandwidth higher than $100 \mathrm{kHz}$ with no saturation in sun exposition, but it could be highly improved if necessary by changing the photosensive element or the operational amplifier. Nevertheless, the useful rate of transmitted data is lower than 100 kbaud in many cases. The second version of the electronics planned o higher gain for tens of meters working distance.

\section{EXPERIMENTAL RESULTS}

In this section, we present the first experimental results that we obtained with the prototype we made. The main objective is to show that the setup is suitable to transmit data using visible light technology. As it has been previously noticed, the emitter and the receiver are microcontrollers and, more precisely, two Microchip PIC18F2550 as they are low cost and widely used microcontrollers. The emitter and the receiver are interfaced with a PC through USB so that we can easily communicate with the microcontrollers.

Basically, the message transmitted during the experiment is sent to the emitter and the frame indicates if Miller or Manchester code is selected. The message is therefore converted into a binary array. The red backlight is then setup to blink periodically according to these values. On the other part, the receiver decodes the data in real-time and an algorithm counts the wrong bits compared to the original message stored in memory.

First results show in Figure 5 that the BER is lower to $3.10^{-5}$ over a distance of few meters. However, it quickly increases when the distance is higher than $10 \mathrm{~m}$. Both curves have been made at $10 \mathrm{kHz}$ modulation frequency, a four synchronization bits configuration and a data length of 4 ascii characters ( $4 * 8$ useful bits). Sets of data are about 3 millions of bits for both configurations. 


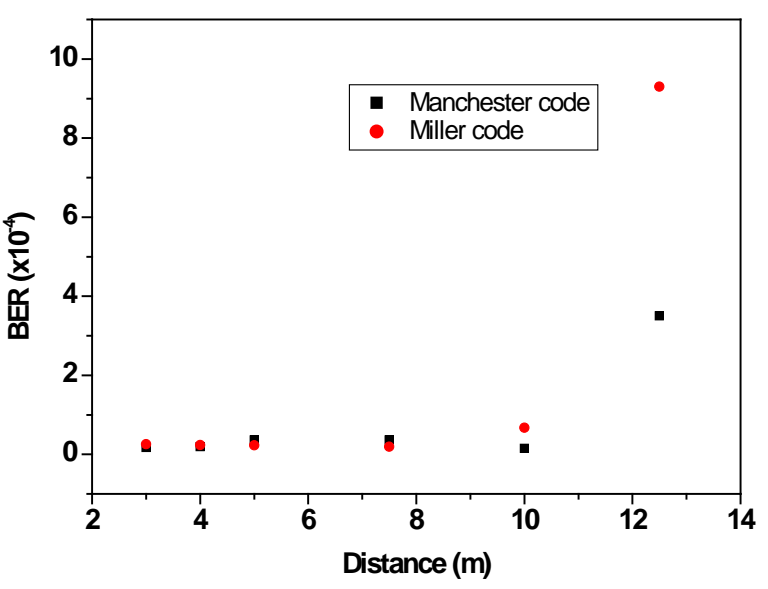

Figure 5: Bit Error Ratio (BER) for Miller and Manchester codes at $10 \mathrm{kHz}$ modulation frequencies.

These results demonstrate that the prototype is well suited to transmit data over a short distance. However, it is a limitation as far as communication between vehicles (e.g. on a motorway) is concerned. One of the main reasons for such a limitation is due to the fact that we intentionally limited the gain. As the purpose of this system is to be used for any weather conditions, we paid attention to select the gain so that the system is not saturated because of the light of the sun. Consequently, the signal to noise ratio decreases as the distance increases. The second main reason is that the clock of the receiver is not synchronized with the transmitted frame. The analog electronics has been aimed to be very simple and no phase locked-loop is included. Nevertheless some analog filters are included that can modify the bit timing, and rise time. The decoding part has an algorithm that is based on count timing with tolerances on the values. But the electronics has to be improved to reduce distortion. A first solution to overcome these problems should be to use an adaptive gain depending on environmental light. The performances would be greatly improved when the light of the sun is moderate. Besides more complex and robust filtering, we also plan to enhance the prototype with an optical filtering based on the color of the light, in order to improve to signal to noise ratio by filtering the useful light. We hope to reach $10^{-6}$ BER over $50 \mathrm{~m}$ distance. First results on the second version of the prototype show a BER less than few $10^{-7}$ on $25 \mathrm{~m}$ distance.

\section{REFERENCES}

[1] C. Canudas de Wit and B. Brogliato, "Stability issues for vehicle platooning in automated highway systems", Proceedings of the IEEE Int. Conf. on Control Applications, vol. 2, 1377-1382, 1999.

[2] T. Komine, J. Hwan Lee, S. Haruyama and M. Nakagawa, "Adaptive Equalization System for Visible Light Wireless Communication Utilizing Multiple White LED Lighting Equipment”, IEEE Transactions on Wireless Communications, vol. 8, no. 6, 2892-2900, 2009.

[3] B. Krogh and C. Thorpe, "Integrated Path Planning and Dynamic Steering Control for Autonomous Vehicles”, Proceedings of the IEEE Int. Conf. on Robotics and Automation, 1664-1669, 1986.

[4] T. Fraichard and J.-M. Ahuactzin. Smooth Path Planning for Cars, Proceedings of the IEEE Int. Conf. on Robotics and Automation, vol.
4, 3722-3727, 2001.

[5] T. Komine and M. Nakagawa, "Fundamental Analysis for VisibleLight Communication System using LED Lights”, IEEE Transactions on Consumer Electronics, Vol. 50, No. 1, 100-107, 2004.

[6] T. Komine and M. Nakagawa, "Integrated System of White LED Visible-Light Communication and Power-Line Communication", IEEE Transactions on Consumer Electronics, vol. 49, no. 1, 71-79, 2003.

[7] M. Wada, T. Yendo, T. Fujii and M. Tanimoto,"Road-to-Vehicle Communication Using LED Traffic Light”, Intelligent Vehicles Symposium, 2005. Proceedings. IEEE, 2005.

[8] N. Kumar, D. terra, N. Lourenço, L.N . Alves, R.L. Aguiar, « Visible light communication for intelligent transportation in road safety applications", Wireless Communications and mobile computing conference (IWCMC), $20117^{\text {th }}$ international, pp. 1513-1518, doi: 10.1109/IWCMC.2011.5982762, 2011.

[9] C. Liu, B. Sadeghi, E.W. knightly, "Enabling vehicular visible light communication (V2LC) networks”, Vanet'11, Las vegas, USA, 2011.

[10] M. Akanegawa, Y. Tanaka and M. Nakagawa, "Basic Study on Traffic Information System Using LED Traffic Lights”, IEEE Transactions on Intelligent Transportation Systems, vol. 2, no. 4, 197-203, 2001.

[11] H. Sing Liu and G. Pang, "Positioning Beacon System Using Digital Camera and LEDs", IEEE Transactions on Vehicular Technology, vol. 52, no. 2, 406-419, 2003.

[12] http://www.nytimes.com/2010/10/10/science/10google.html?_r=3\&p artner=rss\&emc $=$ rss\&pagewanted $=$ all

[13] http://www.sartre-project.eu/en/Sidor/default.aspx

[14] http://www.vlcc.net/?ml_lang=en

[15] http://www.path.berkeley.edu/nahsc/

[16] M. Akanegawa, Y. Tanaka, M. Nakagawa., "Basic Study on Traffic Information System Using LED Traffic Lights”, IEEE Transactions on Intelligent Transportation Systems, Vol. 2, NO. 4, 2001.

[17] T. Nagura, T. Yamazato, M. Katayam, T. Yendo, T. Fujii, H. Okada, "Improved decoding methods of visible lights communication system for ITS using led array and high speed camera”, Vehicular technology conference, VTC 2010 IEEE 71', ISSN: 1550-2252, 2010.

[18] S. Okada, T. Yendo, T. Yamazato, T. Fujii, M. Tanimoto, Y. Kimura, "On-vehicle receiver for distant visible light road-to-vehicle communication”, Intelligent Vehicle Symposium, IEEE ISSN: 19310587, 2009.

[19] Y. Alayli, S. Topsu, L. Chassagne, J.M. Blosseville, Patent n09 58694, Communications par phares, 2009. 